\title{
Translational Control of Gene Expression
}

\author{
MAARTEN JANSEN, CORNELIA H. DE MOOR, JOHN S. SUSSENBACH, AND \\ J. LEO VAN DEN BRANDE \\ Department of Pediatrics and Graduate School for Developmental Biology, Utrecht University, \\ Utrecht, The Netherlands [M.J., C.H.d.M., J.L.V.d.B] and Laboratory for Physiologic Chemistry and \\ Graduate School for Developmental Biology, Utrecht University, Utrecht, The Netherlands [J.S.S.]
}

\begin{abstract}
Translational regulation of mRNA is an important step in the control of gene expression. In a general way, the efficiency of the translational apparatus can be influenced either positively or negatively by changing the level or the activity of rate-limiting protein factors taking part in the process of translation. But translational control can also be very specific, affecting only a single mRNA or class of mRNA molecules. In most of these cases regulation takes place at the level of initiation of translation, which is often attributable to structural peculiarities of the mRNA in question, especially of the $5^{\prime}$-untranslated region or leader. This review summarizes the mechanisms which lie at the root of translational control. A better understanding of these mechanisms will eventually provide us with new drugs and antisense oligonucleotide technology, aimed at influencing the
\end{abstract}

\section{ABSTRACT}

level of expression of single proteins. These developments are of interest to basic researchers and clinicians alike, because they may profoundly change the ways in which we treat, e.g. viral infections and malignancies in the future. (Pediatr Res 37: 681-686, 1995)
eIF, eukaryotic initiation factor
IRE, iron-responsive element
IRES, internal ribosome entry site
BP, binding protein
mRNP, messenger ribonucleoprotein
ORF, open reading frame
TNF, tumor necrosis factor

Regulation of mRNA translation, although still less well characterized than the regulation of gene transcription, is now recognized as one of the major regulatory steps in the control of gene expression (1). In recent years, a variety of ways in which translation of specific mRNA molecules can be regulated have been elucidated. It is now clear that translational control is of paramount importance to the regulation of germ cell differentiation, morphogenesis, the cell cycle and iron metabolism. Translational control can be very specific, aimed at translation of only part of the mRNA in the cell or even a single one. A better understanding of the mechanisms involved in translational control will therefore not only have an impact on basic research but, before long, onto clinical practice as well, as it may aid in the development of new drugs aimed at influencing the level of expression of specific proteins.

This review summarizes the ways in which translational control can be mediated. We will limit the discussion to mechanisms of translational control in a rather strict sense. For a discussion of other principles of posttranscriptional regulation of gene expression, for instance regulation of mRNA

Correspondence and reprint requests: Dr. M. Jansen, Department of Pediatric Endocrinology, Wilhelmina Children's Hospital, P.O. Box 18009, 3501 CA Utrecht, The Netherlands.

Manuscript dedicated to Professor H.K.A. Visser in honor of his retirement. stability, the reader is referred to a number of excellent reviews (2-4).

\section{THE TRANSLATIONAL MACHINERY}

After transcription in the nucleus, a eukaryotic mRNA acquires a cap structure at its $5^{\prime}$-end and a poly(A)-tail at the $3^{\prime}$-end. The RNA strand between these two structures can be thought of as divided into three domains: the $5^{\prime}$-untranslated region or leader, the region encoding the protein, and the $3^{\prime}$-untranslated region or trailer. Of course, in the intact molecule this partition is only artificial: secondary and tertiary interactions take place along the entire strand, within and between these regions. RNA easily forms higher order structures, which as a matter of fact is a prerequisite for the proper fulfilment of its biologic functions (5). A variety of molecules binding to the RNA-strand can change its conformation and this in turn can be expected to have an impact on translation.

Cytoplasmic mRNA molecules are either actively translated by ribosomes with efficiencies that may differ for specific classes of mRNA, or they are translationally repressed, i.e. associated with proteins in the form of messenger ribonucleoprotein particles, mRNP $(6,7)$. To become translated, mRNA molecules must be recruited, or mobilized, from the pool of mRNP. Once mobilized, the mRNA can be translated, a pro- 
cess that can be divided basically into three phases: initiation, elongation, and termination.

The goal of initiation is to position the ribosome at the start of the coding region; this phase ends with the formation of the first peptide bond. For most mRNA species the search for the coding region starts at the cap, which is recognized by the cap binding initiation factor eIF-4E. This protein is found in a complex with other initiation factors, among which an RNAhelicase (8). The latter starts unwinding the RNA leader, which enables the so-called initiation complex, composed of the small ribosomal subunit, methionyl-tRNA and the initiation factor eIF2, to begin scanning for the coding region (9). The start of the coding region is marked by an initiation codon, generally an AUG (which encodes methionine). Once the scanning complex encounters a proper initiation codon, it is lined up with the methionyl-tRNA on the small subunit, after which the large ribosomal subunit joins the complex. Elongation of the peptide chain then begins, catalyzed by a domain on the large ribosomal subunit and facilitated by yet another protein, elongation factor $1(10)$. The actual processes taking place during elongation, the codon-by-codon decoding of the mRNA and peptide bond formation, proceed at an amazing speed of easily more than 5 aminoacyl residues per second. Finally, termination occurs when a termination codon (UAA, UAG, or UGA) arrives in the active ribosomal site. At that point, the peptide chain and the mRNA are released from the ribosome (11), ignoring the remainder of the mRNA strand.

\section{GENERAL ASPECTS OF TRANSLATIONAL REGULATION}

Translation takes place in the cytoplasm. Some parts of the cytoplasm, however, are so tightly packed with soluble protein and cytoskeleton that ribosomes can be expected to have difficulties diffusing into them. Moreover, some components of the translational machinery are themselves associated with the cytoskeleton (12). It is thus entirely possible that local differences in the activity and composition of the translational machinery exist within the cell. Next to the translational repression by proteins binding to the RNA as mentioned above, this may explain how certain mRNA molecules can (temporarily) be sequestered and kept out of translation, as appears to be the case with a major part of one of the mRNA species of human IGF-II (13).

Regulation of the rate of protein synthesis occurs by influencing rate-limiting steps of the translational process. This can be accomplished by modulation of the available amount of ribosomes or initiation factors (14) or, more often, by a change in the activity of these factors through phosphorylation or dephosphorylation $(1,14)$. The initiation factor eIF-2, for instance, becomes phosphorylated on its $\alpha$-subunit in response to a variety of cellular emergencies such as heat shock, hypertonicity and viral infection, and this severely inhibits protein synthesis. Phosphorylation of most other initiation factors, in contrast, correlates with improved protein synthesis (15).

In case of translational regulation of only one or a small class of mRNA molecules other determinants come into play, such as the available amount of the mRNA in question, as determined by its rate of transcription, processing and turnover, or structural aspects of that particular mRNA or class of mRNA molecules. Almost invariably, regulation at the level of initiation is observed in these cases. In most cells, the availability of the cap-binding protein eIF-4E is the rate-limiting factor in translation initiation (1). Regulation of eIF-4E levels or activity appears therefore a very efficient tool to control the rate of translation, especially of those mRNA transcripts that have difficulties in being translated anyway due to structural peculiarities of their leaders. We come back to this later.

An increasing number of trans-acting factors, commonly proteins associated with mRNA in the messenger ribonucleoprotein particles, are now being recognized as modulators of translation. Many of these proteins and their RNA-binding domains have already been characterized (16). The specificity of binding of these proteins varies and in many cases it is not clear whether binding truly modulates translation. It is conceivable that at least some of them play a role in changing the rate of translation of the mRNA molecules to which they bind.

The following main targets of translational control can thus be identified (1) (Fig. 1): 1) Mobilization i.e. the shifting of mRNA between the mRNP fraction and polysomes, as occurs for instance with insulin mRNA in islet cells under highglucose conditions (17); trans-acting factors appear to play a major role in this process. 2) Alteration of initiation rates, influenced mainly by the availability and activity of translation initiation factors and by cis-acting elements, i.e. structural peculiarities of the mRNA in question. Typically these are located in the leader or trailer of the mRNA strand. 3) Modulation of elongation and termination rates appear to be less important in translational control, but frame-shifts and stop codon read-throughs can be used by cis-elements in the coding region to regulate gene expression. They will be discussed only briefly. 4) Elements involved in regulation of mRNA stability are frequently contained within the $3^{\prime}$-untranslated region of mRNA transcripts; as noted in the introduction, this aspect of the control of gene expression is beyond the scope of this review.

\section{LEADER-MEDIATED TRANSLATIONAL CONTROL}

Because the $5^{\prime}$-untranslated region of an mRNA has to be passed by the scanning ribosome, this is the obvious target for regulating translational efficiency. By definition, the leader ends where the coding region begins and translation starts. As

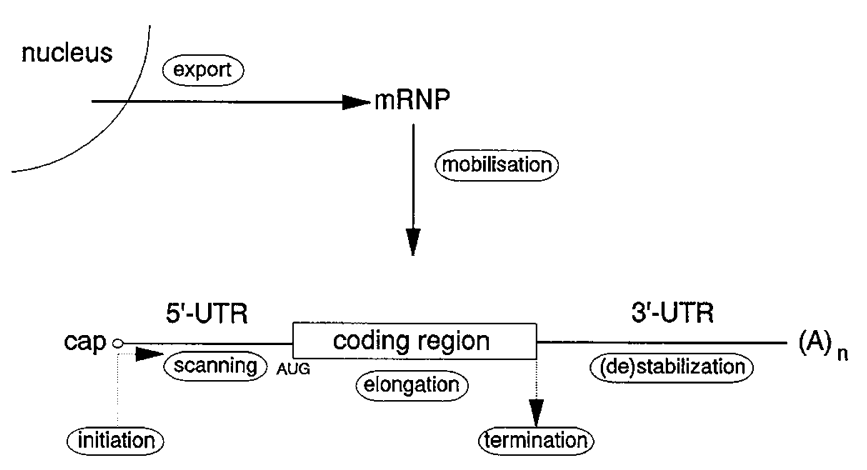

Figure 1. Targets of translational regulation. 
discussed above, in the vast majority of cases this is at the first AUG encountered by the initiation complex. To be recognized, this initiator AUG must be in a favorable context, ideally CCPCCAUGG, in which $\mathrm{P}$ is a purine $(18,19)$. Moreover, it must be localized at a sufficient distance from the cap (20). If AUG codons are present in the leader upstream of the genuine initiation codon of the encoded protein, this can severely inhibit translation, especially if these AUGs are in a favorable context. The scanning ribosomes initiating at these "false" initiation codons usually cannot reinitiate at the genuine initiation codon.

An ideally efficient RNA would thus have a leader of moderate length, devoid of secondary structure and upstream initiation codons, followed by an AUG codon in a favorable context. Most proteins that are highly expressed have mRNA molecules that come close to this ideal. Leaders of mRNA molecules encoding proteins with a strictly regulated expression, however, are often much longer than the average of 100-140 nucleotides $(18,19)$, contain upstream AUG codons and/or are expected to form stable secondary structures by virtue of a high $\mathrm{G}+\mathrm{C}$ content $(21,22)$. A large number of mRNA molecules for proteins involved in growth and development fall in this category. Many of these long leaders have been shown to be detrimental to translation (23).

How then can the translational efficiency of these "difficult" leaders containing a lot of secondary structure be improved? One way is by changing the levels or the activity of the limiting factor for translation initiation, eIF-4E, so that even these highly structured leaders get a chance of entering translation (24). The translation of natural mRNA transcripts containing structured leaders, such as ornithine aminotransferase and growth regulating proteins like cyclin D1 and transcription factors, can indeed be induced by eIF-4E overexpression (26) and this is probably how overexpression or enhanced activity of eIF-4E may lead to oncogenic transformation $(26,27)$.

Another way by which translation of such mRNA transcripts can be improved is by proteins that bind specifically to the leader. Such proteins can influence the structure of the leader, stabilizing, destabilizing, or otherwise altering its conformation. Alternatively, a tightly bound RNA BP can mimick secondary structure by obscuring the cap or stalling the scanning complex in its early stages, thereby hampering translation. The prototype of mRNA-specific translational control by RNA $\mathrm{BP}$ is the translational regulation of ferritin expression by iron (28). The leader of ferritin mRNA contains a region of approximately 30 nucleotides that can fold into a stem-loop structure, the iron-response element or IRE. When cellular iron levels are low, a protein binds to this region (the IRE BP). This causes a repression of ferritin-mRNA translation. This makes sense, because there is little need for ferritin under these circumstances. But as soon as cellular iron levels increase, the IRE BP is displaced from its binding site either by iron itself or by hemin (29), so that translation of ferritin mRNA can take place. Most remarkably, the mRNA of another iron-regulating protein, the transferrin receptor, contains five IRE in its $3^{\prime}$ untranslated region, to which IRE BP can bind if cellular iron concentrations are high. This destabilizes the messenger, leading to its increased degradation. The resultant reduction in transferrin receptor expression at the cell surface then limits further iron uptake by the cell (30). Several other mRNA molecules have since been shown to contain an IRE, such as the messengers for 5-aminolevulinate synthetase and aconitase $(31,32)$. Recently, the second messenger nitric oxide (NO) was shown to be able to regulate the RNA-binding activity of IRE $\mathrm{BP}$ (33) but the physiologic significance of this phenomenon is not yet clear.

Other examples of translational regulation by proteins binding to RNA are now emerging, such as the autoregulation of thymidilate synthetase and dihydrofolate reductase activity which may have implications for the design of anti-tumor drugs (34), the regulation of transforming growth factor $\beta 1$ synthesis (35) and the relief of translational inhibition by binding of the Tat-protein of human immunodeficiency virus to its RNA-binding site, TAR (36). We have recently been able to demonstrate stimulation of translation in vitro by proteins binding specifically to a circumscript region in one of the four leaders (L3) of IGF-II mRNA; one of these proteins appears to be the $57-\mathrm{kD}$ polypyrimidine tract $\mathrm{BP}$ (37).

As discussed above, upstream AUG codons or ORF can be strong repressors of translation. There are two ways in which this repression can be mitigated: 1) The scanning complex may not always recognize the upstream AUG, especially if it has a suboptimal context, resulting in continued scanning for the next initiation codon. This is called "leaky scanning." 2) After translation of a small ORF the ribosome is sometimes able to resume scanning for a second AUG. This has only been reported for small ORF and is called resumed scanning or reinitiation.

Normally, neither leaky scanning nor reinitiation is very efficient (38): many mRNA species encoding proteins involved in growth and development contain an upstream ORF which may contribute to their inefficient translation. There are, however, a few cases of cellular mRNA species in which an upstream ORF forms an integral part of a translational regulation mechanism. A beautiful example is the mRNA encoding the $\beta_{2}$-adrenergic receptor, which harbors a short ORF in its leader; the 19-amino acid peptide encoded by this ORF has been shown to inhibit translation of the receptor mRNA (39). A similar mechanism has been proposed for the hexapeptide encoded by a $5^{\prime}$-leader ORF in the mRNA of $S$-adenosylmethionine decarboxylase (40).

The translation of the vast majority of mRNA transcripts depends on the presence of a cap and involves scanning of the whole leader. Yet the mRNA of picorna viruses, although uncapped and endowed with long, highly structured leaders that are riddled with upstream ORF, are translated quite efficiently. These mRNA transcripts initiate via a cap-independent mechanism called internal initiation $(41,42)$. Here, the initiation complex enters the leader in an IRES instead of at the 5 '-end. A genuine IRES will thus direct translation of a downstream ORF, regardless of the secondary structure or upstream ORF that precede it.

The picorna IRES share little sequence homology except for a polypyrimidine tract located approximately 20 nucleotides from the initiation codon, which is essential for internal initiation (43). Two cellular proteins have been shown to be 
involved in internal initiation, La protein and polypyrimidine tract BP. Both are primarily nuclear proteins but appear to play a role in translation as well, because their immunodepletion from in vitro translation systems inhibits internal initiation (43). Because the viral proteins per se play no part in internal initiation, any cellular mRNA with the appropriate structure should be able to translate by internal initiation. The mRNA encoding human immunoglobulin heavy chain binding protein (BiP) was the first cellular mRNA for which translation by internal initiation was reported (44). Recently, internal initiation was also demonstrated to occur in vitro in the leader of the adult liver type mRNA of IGF-II mRNA (L1) (45). A computer-fold of this leader indeed shows an IRES-like "platform" of single-stranded nucleotides, as in the BiP and picorna virus leaders (Thomas A, personal communication). No sequence homologies between the putative IGF-II IRES, the BiP IRES, and the picorna virus IRES have been identified (44).

\section{TRANSLATIONAL CONTROL MEDIATED BY THE CODING REGION}

Apart from recognition of the initiation codon, regulation of translation by sequences in the coding region is not a widespread phenomenon. Although some coding regions contain "ribosomal pause" sites, probably caused by secondary structure in the coding region or by codons requiring rare or depleted aminoacyl-tRNAs, the elongation rate under nonstarvation conditions is considered to be near-maximal. An exception is the increase in elongation rate of the heat shock protein HSP70 during heat shock, both in vivo and in vitro (46).

A growing number of eukaryotic genes, among which basic fibroblast growth factor and several mammalian transcription factors such as Pit-1 and c-myc, employ multiple initiation codons to produce $\mathrm{N}$-terminally extended or truncated protein products (47-49). This probably occurs by leaky scanning. Regulation of the ratio of the products of alternative initiation has been reported, for instance for c-myc mRNA (49). Although this regulation is still poorly understood, it may have profound biologic consequences because of the differences between the biologic activities of these products.

Some sequences in ORF can induce a frameshift at a specific codon with a high frequency. This is frequently found in viral genomes (50), but it has also been discovered in a mammalian mRNA, ornithine decarboxylase antizyme (51). This protein functions as a repressor of ornithine decarboxylase, the ratelimiting enzyme of polyamine synthesis. The normal in-frame translation of ornithine decarboxylase antizyme leads to a premature stop, but in the presence of polyamines this premature stop is bypassed by a +1 frameshift.

\section{TRAILER AND POLY(A) TAIL-MEDIATED TRANSLATIONAL CONTROL}

The $3^{\prime}$-untranslated region or trailer is often the largest part of an mRNA. Yet it has long been considered a meaningless link between the stop codon and the poly(A) signal. In the last several years this region has turned out to be a treasure trove of posttranscriptional regulation. RNA processing, export from the nucleus, mRNA stability, and intracellular localization and translation can all be regulated by the trailer $(52,53)$. Recently, the importance of this region was underscored by the discovery that the trailers of muscle structural mRNA molecules can induce differentiation and suppress oncogenicity in myogenic cells, as well as suppress proliferation of fibroblasts, all without the benefit of a coding region (54).

All eukaryotic cellular mRNA transcripts, with the exception of histone messengers, are provided with a poly(A) tail in the nucleus. After entering the cytoplasm, the poly(A) tail is progressively shortened as the mRNA ages. Some very unstable mRNA species, such as those encoding the myc and fos proto-oncogenes, are deadenylated far more rapidly than the average mRNA (55). A wealth of evidence indicates that, next to having a stabilizing influence on mRNA, long poly(A) tails are stimulatory for translation, both in vivo and in vitro (56). A trans-acting factor, the poly (A) tail $\mathrm{BP}$, is involved in this stimulation $(57,58)$. Surprisingly, the poly(A)-poly(A) tail BP complex influences an event taking place far upstream in the mRNA-molecule: the binding of the large ribosomal subunit to the small subunit at the initiation codon (58). The mechanism by which this is achieved is still incompletely understood.

The length of the poly(A) tail can also be increased in the cytoplasm. This occurs for example during early oocyte development in a large number of animals, including Xenopus laevis and the mouse. Some of the maternal mRNA transcripts in the oocytes of these animals are initially not translated, but become activated, or "unmasked," at later stages of oocyte maturation or early embryogenesis. This is accompanied by an increase in the length of their poly $(\mathrm{A})$ tails $(59,60)$. The signal for this cytoplasmic polyadenylation is located in the trailer and consists of the normal polyadenylation signal, AAUAAA and a U-rich sequence located upstream of it $(52,60)$. So far, translational activation by cytoplasmic polyadenylation has been demonstrated only in germ cells, but somatic cells as well do have some adenylation activity in their cytoplasm (61). Interestingly, in two somatic mRNA species, encoding the neuropeptides vasopressin and oxytocin, a strong correlation has been found between induction of peptide production and extension of the poly(A) tail. Whether this coincides with translational activation is still unresolved (62).

Other forms of translational stimulation by trailer elements are as yet poorly understood. Interestingly, a form of the lysosomal storage disease aspartylglucosaminuria has been described resulting from a homozygous deletion of most of the trailer part of the aspartylglucosaminidase mRNA. This deletion caused the synthesis of a stable, truncated but polyadenylated mRNA with a completely intact coding region, which was nevertheless untranslated in vivo, whereas no defect in translational efficiency could be demonstrated in vitro (63). Another example is provided by the mRNA for the amyloid protein precursor, which has two forms produced by alternative polyadenylation. The longer mRNA translates more efficiently than the shorter one, and the available evidence indicates that the region between the two polyadenylation sites is stimulatory for translation (64).

In contrast to translational stimulation by the trailer, the number of mRNA transcripts that is translationally repressed by cis-acting elements in their trailers is expanding and by no 
means restricted to germ cell systems. Such repression can be conferred by AU-rich elements, for instance in the trailers of certain cytokines, human interferon $\beta$, granulocyte-macrophage colony-stimulating factor and c-fos $(65,66)$. Translational control of another cytokine, TNF, takes part in the upregulation of TNF production in macrophages in response to bacterial endotoxin (67). Here also, the translational regulation is conferred by the trailer and the AU-rich element is essential both for repression in nonactivated cells and derepression in activated cells. Dexamethasone suppresses TNF translation, which is also mediated by the trailer (68). Translational repression can also be conferred by proteins binding to the trailer, e.g. in the case of protamine mRNA during spermatid maturation (52) or of 15-lipoxygenase mRNA during reticulocyte maturation (69).

\section{CONCLUDING REMARKS}

This overview of the known mechanisms of mRNA-specific translational control illustrates both the great diversity of translational regulation and the essentially limited number of steps that are affected. Most of the examples given were disclosed during the last 6 or $7 \mathrm{y}$, and the number of mRNA molecules subject to translational control is still rapidly increasing. Although many of the mechanisms involved are still far from being completely understood, the principles of translational regulation will soon find their way into basic research as well as clinical practice.

In research, for instance, the efficiency of recombinant protein biosynthesis can be improved by clever engineering of expression plasmids, e.g. by optimizing the length and composition of leaders and trailers, the context of the initiation codon, and the stability of the transcript. Elements such as the IRE in ferritin mRNA will provide constructs with a molecular switch mechanism enabling the voluntary expression of the protein under study, and a further characterization of elements conferring tissue and/or development-specific regulation at the translational level will lead to additional refinements in expression patterns, e.g. in transgenic animals.

In clinical practice, the ability to interfere with translational processes may completely change the ways in which we treat viral and malignant diseases (70). Clinical trials with antisense oligonucleotides have already started, aimed at blocking translation of very specific classes of mRNA, be they viral, bacterial or aberrant human ones. By binding to the mRNA in question they may directly obstruct translation, e.g. by interfering with cap recognition or RNA unwinding, or they may prevent the binding of trans-acting factors that modulate translation. In addition, they can stimulate endonucleolytic cleavage and destruction of the mRNA. This is a highly promising novel way of treating viral infections (e.g. human immunodeficiency virus) and malignancies, especially if used as an adjunct to the regular antiviral and cytostatic drugs. Without being unrealistic, a substantial refinement of our therapeutic possibilities in nonmalignant growth disturbances and many viral and genetic diseases can be expected to result from a further increase in our knowledge of the mechanisms of translational control.

\section{REFERENCES}

1. Hershey JWB 1991 Translational control in mammalian cells. Annu Rev Biochem 60:717-755

2. Hentze MW 1991 Determinants and regulation of cytoplasmic mRNA stability in eukaryotic cells. Biochim Biophys Acta 1090:281-292

3. Peltz SW, Brewer G, Bernstein P, Hart PA, Ross J 1991 Regulation of mRNA turnover in eukaryotic cells. Crit Rev Eukaryotic Gene Expression 1:99-126

4. Peltz SW, Jacobson A 1992 mRNA stability: in trans-it. Curr Opin Cell Biol 4:979-983

5. Moore PB 1993 Recent RNA structures. Curr Opin Struct Biol 3:340-344

6. De Moor CH, Van Heugten HAA, Voorma HO 1990 Characterization of messenger ribonucleoprotein particles. Mol Biol Rep 14:57-60

7. Spirin AS 1994 Storage of messenger RNA in eukaryotes: envelopment with protein, translational barrier at $5^{\prime}$ side, or conformational masking by $3^{\prime}$ side? Mol Reprod Dev 38:107-117

8. Fuller-Pace FV 1994 RNA helicases: modulators of RNA structure. Trends Cell Biol 4:271-274

9. Kozak M 1989 The scanning model for translation: an update. J Cell Biol 108:229241

10. Proud CG 1994 Peptide-chain elongation in eukaryotes. Mol Biol Rep 19:161-170

11. Tuite MF, Stansfield I 1994 Termination of protein synthesis. Mol Biol Rep 19:171181

12. Hesketh J 1994 Translation and the cytoskeleton: a mechanism for targeted protein synthesis. Mol Biol Rep 19:233-243

13. De Moor CH, Jansen M, Sussenbach JS, Van den Brande JL 1994 Differential polysomal localization of human insulin-like growth factor 2 mRNAs, both in cell lines and foetal liver. Eur J Biochem 222:1017-1022

14. Rhoads RE 1993 Regulation of eukaryotic protein synthesis by initiation factors. J Biol Chem 268:3017-3020

15. Pain VM 1986 Initiation of protein synthesis in mammalian cells. Biochem $J$ 235:625-637

16. Mattaj IW 1993 RNA recognition: a family matter. Cell 73:837-840

17. Welsh M, Scherberg N, Gilmore R, Steiner DF 1986 Translational control of insulin biosynthesis. Biochem J 235:459-467

18. Kozak M 1987 An analysis of $5^{\prime}$ noncoding sequences from 699 vertebrate messenger RNAs. Nucleic Acids Res 15:8125-8148

19. Pesole G, Fiormarino G, Saccone C 1994 Sequence analysis and compositional properties of untranslated regions of human mRNAs. Gene 140:219-225

20. Kozak M 1991 A short leader sequence impairs the fidelity of initiation by eukaryotic ribosomes. Gene Expression 1:111-115

21. Kozak M 1989 Circumstances and mechanisms of inhibition of translation by secondary structure in eukaryotic mRNAs. Mol Cell Biol 9:5134-5142

22. Gray NK, Hentze MW 1994 Regulation of protein synthesis by mRNA structure. Mol Biol Rep 19:195-200

23. Kozak M 1991 An analysis of vertebrate mRNA sequences: intimations of translational control. J Cell Biol 115:887-903

24. Koromilas AE, Lazaris-Karatzas A, Sonenberg N 1992 mRNAs containing extensive secondary structure in their $5^{\prime}$ non-coding region translate efficiently in cells overexpressing initiation factor eIF-4E. EMBO J. 11:4153-4158

25. Fagan RJ, Lazaris-Karatzas A, Sonenberg N, Rozen R 1991 Translational control of ornithine aminotransferase. Modulation by initiation factor eIF-4E. J Biol Chem 266:16518-16523

26. Rosenwald IB, Lazaris-Karatzas A, Sonenberg N, Schmidt EV 1993 Elevated levels of cyclin $\mathrm{D} 1$ protein in response to increased expression of eukaryotic initiation factor 4E. Mol Cell Biol 13:7358-7363

27. Barve SS, Cohen DA, De Benedetti A, Rhoads RE, Kaplan AM 1994 Mechanism of differential regulation of IL-2 in murine Th1 and Th2 T cell subsets: 1 . Induction of IL-2 transcription in Th2 cells by up-regulation of transcription factors with the protein synthesis initiation factor 4E. J Immunol 152:1171-1181

28. Melefors Ö, Hentze MW 1993 Translational regulation by mRNA/protein interactions in eukaryotic cells: ferritin and beyond. BioEssays 15:85-90

29. Lin J-J, Daniels-McQueen S, Patino MM, Gaffield L, Walden WE, Thach RE 1990 Derepression of ferritin messenger RNA translation by hemin in vitro. Science 247:74-77

30. Klausner RD, Rouault TA, Harford JB 1993 Regulating the fate of mRNA: the control of cellular iron metabolism. Cell 72:19-28

31. Dandekar T, Stripecke R, Gray NK, Goossen B, Constable A, Johansson HE, Hentze MW 1991 Identification of a novel iron-responsive element in murine and human erythroid $\delta$-aminolevulinic acid synthase mRNA. EMBO J 10:903-1909

32. Haile DJ, Rouault TA, Tang CK, Chin J, Harford JB, Klausner RD 1992 Reciprocal control of RNA-binding and aconitase activity in the regulation of the iron-responsive element binding protein: role of the iron-sulfur cluster. Proc Natl Acad Sci USA 89:7536-7540

33. Weiss G, Goossen B, Doppler W, Fuchs D, Pantopoulos K, Werner-Felmayer G, Wachter H, Hentze MW 1993 Translational regulation via iron-responsive elements by the nitric oxide/NO-synthase pathway. EMBO J 12:3651-3657

34. Chu E, Takimoto CH, Voeller D, Grem JL, Allegra CJ 1993 Specific binding of human dihydrofolate reductase protein to dihydrofolate reductase messenger RNA in vitro. Biochemistry 32:4756-4760

35. Kim S-J, Park K, Koeller D, Kim KY, Wakefield LM, Sporn MB, Roberts AB 1992 Post-transcriptional regulation of the human transforming growth factor- $\beta 1$ gene. J Biol Chem 267:13702-13707

36. Cullen BR 1990 The HIV-1 Tat protein: an RNA sequence-specific processivity factor. Cell 63:655-657 
37. De Moor CH, Jansen M, Bonte EJ, Thomas AAM, Sussenbach JS, Van den Brande JL 1994 Influence of the four leaders of the human insulin-like growth factor $r$ mRNAs on the expression of reporter genes. Eur J Biochem 226:1039-1047

38. Kozak M 1992 Regulation of translation in eukaryotic systems. Annu Rev Cell Biol 8:197-225

39. Parola L, Kobilka BK 1994 The peptide product of a $5^{\prime}$ leader cistron in the $\beta_{2}$ adrenergic receptor mRNA inhibits receptor synthesis. J Biol Chem 269:4497-4505

40. Hill JR, Morris DR 1992 Cell-specific translation of $S$-adenosylmethionine decarboxylase mRNA. Regulation by the $5^{\prime}$ transcript leader. J Biol Chem 267:21886-21893

41. Pelletier J, Sonenberg N 1988 Internal initiation of translation of eukaryotic mRNA directed by a sequence derived from poliovirus RNA. Nature 334:320-325

42. Jackson RJ, Hunt SL, Gibbs CL, Kaminski A 1994 Internal initiation of translation of picornavirus RNAs. Mol Biol Rep 19:147-159

43. Hellen CUT, Witherell GW, Schmid M, Shin SH, Pestova TV, Gil A, Wimmer E 1993 A cytoplasmic $57-\mathrm{kDa}$ protein that is required for translation of picornavirus RNA by internal ribosomal entry is identical to the nuclear pyrimidine tract-binding protein. Proc Natl Acad Sci USA 90:7642-7646

44. Macejak DG, Sarnow P 1991 Internal initiation of translation mediated by the 5' leader of a cellular mRNA. Nature 353:90-94

45. Teerink H, Kasperaitis MAM, De Moor CH, Voorma HO, Thomas AM 1994 Translation initiation on the insulin-like growth factor II leader 1 is developmentally regulated. Biochem J 303:547-553

46. Theodorakis NG, Baneiji SS, Morimoto RI 1988 HSP70 mRNA translation in chicken reticulocytes is regulated at the level of elongation. J Biol Chem 263:14579-14585

47. Voss JW, Yao T-P, Rosenfeld MG 1991 Alternative translation initiation site usage results in two structurally distinct forms of Pit-1. J Biol Chem 266:12832-12835

48. Bugler B, Amalric F, Prats H 1991 Alternative initiation of translation determines cytoplasmic or nuclear localization of basic fibroblast growth factor. Mol Cell Biol 11:573-577

49. Hann SR, Sloan-Brown K, Spotts GD 1992 Translational activation of the non-AUGinitiated c-myc 1 protein at high cell densities due to methionine deprivation. Genes Dev 6:1229-1240

50. Brierley I 1993 Probing the mechanism of ribosomal frameshifting on viral RNAs. Biochem Soc Trans 21:822--826

51. Rom E, Kahana C 1994 Polyamines regulate the expression of ornithine decarboxylase antizyme in vitro by inducing ribosomal frame-shifting. Proc Natl Acad Sci USA 91:3959-3963

52. Jackson RJ 1993 Cytoplasmic regulation of mRNA function: the importance of the 3 untranslated region. Cell 74:9-14

53. Theil EC 1993 The IRE (iron regulatory element) family: structures which regulate mRNA translation or stability. BioFactors 4:87--93
54. Rastinejad F, Blau HM 1993 Genetic complementation reveals a novel regulatory role for $3^{\prime}$ untranslated regions in growth and differentiation. Cell 72:903-917

55. Schiavi SC, Belasco JG, Greenberg ME 1992 Regulation of proto-oncogene mRNA stability. Biochim Biophys Acta Rev Cancer 1114:95-106

56. Munroe D, Jacobson A 1990 Tales of poly(A): a review. Gene 91:151-158

57. Grossi De Sa M-F, Standart N, Martins de Sa C, Akhayat O, Huesca M, Scherrer K 1988 The poly(A)-binding protein facilitates in vitro translation of poly(A)-rich mRNA. Eur J Biochem 176:521-526

58. Munroe D, Jacobson A 1990 mRNA poly(A) tail, a $3^{\prime}$ enhancer of translational initiation. Mol Cell Biol 10:3441-3455

59. Bachvarova RF 1992 A maternal tail of poly(A): the long and the short of it. Cell 69:895-897

60. Richter JD 1993 Translational control in development: a perspective. Dev Genet 14:407-411

61. Jackson RJ, Standart N 1990 Do the poly(A) tail and $3^{\prime}$ untranslated region control mRNA translation. Cell 62:15-24

62. Robinson BG, Frim DM, Schwartz WJ, Majzoub JA 1988 Vasopressin mRNA in the suprachiasmatic nuclei: daily regulation of polyadenylate tail length. Science 241:342-344

63. Ikonen E, Ulmanen I, Peltonen L 1992 Deletion of the 3'-untranslated region of aspartylglucosaminidase mRNA results in a lysosomal accumulation disease. $\mathrm{J}$ Biol Chem 267:8715-8718

64. De Sauvage F, Kruys V, Marinx O, Huez G, Octave JN 1992 Alternative polyadenylation of the amyloid protein precursor mRNA regulates translation. EMBO J 11:3099-3103

65. Kruys V, Marinx O, Shaw G, Deschamps J, Huez G 1989 Translational blockade imposed by cytokine-derived UA-rich sequences. Science 245:852-855

66. Grafi G, Sela I, Galili G 1993 Translational regulation of human beta interferon mRNA: Association of the $3^{\prime}$ AU-rich sequence with the poly(A) tail reduces translation efficiency in vitro. Mol Cell Biol 13:3487-3493

67. Han J, Huez G, Beutler B 1991 Interactive effects of the tumor necrosis factor promoter and $3^{\prime}$-untranslated regions. J Immunol 146:1843-1848

68. Beutler B 1992 Application of transcriptional and posttranscriptional reporter constructs to the analysis of tumor necrosis factor gene regulation. Am J Med Sci 303:129-133

69. Ostareck-Lederer A, Ostareck DH, Standart N, Thiele BJ 1994 Translation of 15-lipoxygenase mRNA is inhibited by a protein that binds to a repeated sequence in the $3^{\prime}$ untranslated region. EMBO J 13:1476-1481

70. Cohen JS, Hogan ME 1994 The new genetic

\section{Erratum}

In the article "Deconvolution Analysis of Spontaneous Nocturnal Growth Hormone Secretion in Prepubertal Children with Preterminal Chronic Renal Failure and with End-Stage Renal Disease" by Burkhard Tönshoff et al. (Pediatr Res 37:86-93, 1995) each value in " $\mathrm{mg} / \mathrm{L}$ " should be " $\mu \mathrm{g} / \mathrm{L}$ ". In addition, in Table 2 , "GH production $/ \mathrm{m}^{2}$ body surface area $\left(\mathrm{mg} / \mathrm{m}^{2} / 10 \mathrm{~h}\right)$ " should be "GH production $/ \mathrm{m}^{2}$ body surface area $\left(\mu \mathrm{g} / \mathrm{m}^{2} / 10 \mathrm{~h}\right)$ ". The authors regret this error. 